\title{
Uso de escoria de cobre de un vertedero abandonado en mezclas asfálticas
}

\author{
Use of copper slag from an abandoned landfill in asphalt mixes
}

Fecha de entrega: 27 de diciembre 2020

Fecha de aceptación: 3 de septiembre 2021

\begin{abstract}
Amin Nazer ${ }^{1,2}$, María Castillo ${ }^{1}$, Alicia López ${ }^{1}$, Leonel Ortiz ${ }^{3}$ y Osvaldo Pavez $^{2,4}$

${ }^{1}$ Departamento de Construcción, Facultad Tecnológica, Universidad de Atacama, Avenida Copayapu 485, Copiapó, Chile, amin.nazer@uda.cl,maria.castillo@alumnos.uda.cl,alicia.lopez@alumnos.uda.cl

${ }^{2}$ Centro Regional de Investigación y Desarrollo Sustentable de Atacama CRIDESAT, Universidad de Atacama, Avenida Copayapu 485, Copiapó, Chile, osvaldo.pavez@uda.cl

${ }^{3}$ Laboratorio Regional de Vialidad, Tierra Amarilla, Chile, leonel.ortiz@vialidad.mop.cl

${ }^{4}$ Departamento de Ingeniería en Metalurgia, Facultad de Ingeniería, Universidad de Atacama, Avenida Copayapu 485, Copiapó, Chile
\end{abstract}

La escoria de cobre se considera un pasivo ambiental minero y normalmente se deposita en vertederos. Sin embargo, algunas investigaciones se han orientado a su utilización en la fabricación de morteros y hormigones. Por otra parte, no se han reportado estudios sobre el uso de escorias de cobre de vertederos abandonados del siglo XIX en el diseño de pavimentos asfálticos. En este estudio se evaluó el comportamiento de mezclas asfálticas en caliente conteniendo escoria de cobre de un vertedero abandonado del norte de Chile, como un sustituto parcial del árido grueso. Para el diseño y control de mezclas de pavimento bituminoso se utilizó el método de Marshall evaluando la densidad, porcentaje de huecos de aire en la mezcla, porcentaje de vacios en el agregado mineral, fluencia $y$ estabilidad. Además, se evaluaron mezclas asfálticas conteniendo entre 4.5 a $6.0 \%$ de asfalto con incrementos de $0.5 \%$ para cuatro tipos de mezclas con sustitución parcial de árido grueso convencional por escoria de cobre (0, 30, 50 y 70\%, en peso). Como conclusión se puede señalar que, la mezcla con $5 \%$ de asfalto y $30 \%$ de escoria de cobre en remplazo de los áridos, es la mezcla más adecuada y podría ser usada en el diseño de mezclas asfálticas para pavimentos. La utilización de escoria de cobre de vertederos abandonados en la fabricación de pavimentos asfálticos contribuiría a mejorar las condiciones ambientales disminuyendo, además, la demanda de áridos en zonas desérticas como la Región de Atacama en Chile.

Palabras clave: escorias de cobre, pasivo ambiental minero, mezclas asfálticas, método de Marshall
Copper slag is considered a mining environmental liability and is usually deposited in landfills. However, some investigations have been oriented to their use in mortars and concretes. On the other hand, no studies have been reported on the use of copper slag from abandoned landfills of the 19th century in the design of asphalt pavements. In this study, the behaviour of hot asphalt mixtures containing copper slag from abandoned landfill in northern Chile, as a partial substitute for coarse aggregate was evaluated. For the design and control of bituminous pavement mixtures, the Marshall method was used. The parameters evaluated were density, \% air voids in the mixture, \% voids in the mineral aggregate, creep and stability. In addition, asphalt mixtures containing 4.5 to $6.0 \%$ were evaluated with increments of $0.5 \%$ asphalt for four types of mixtures with partial substitution of conventional aggregates by copper slag (0, 30, 50 and $70 \%$, by weight). In conclusion, it can be pointed out that the mixture with $5 \%$ asphalt and 30\% copper slag in replacement of the aggregate is the most suitable mixture and could be used in the design of asphalt mixtures for pavements. The use of copper slag from abandoned landfills in the manufacture of asphalt pavements, would contribute to improving environmental conditions, also decreasing the demand for aggregates in desert areas such as the Atacama Region in Chile.

Keywords: copper slag, mining environmental passive, asphalt mixtures, Marshall method 


\section{Introducción}

La escoria de cobre (EC) se considera un pasivo ambiental minero y normalmente se deposita en vertederos, sin tener un uso alternativo. En el siglo XIX se establecieron alrededor de 40 fundiciones de cobre en el norte de Chile, las cuales produjeron barras de cobre para su exportación, generando EC como un residuo industrial sin valor comercial. El vertido de la EC se realizaba en playas, sectores agrícolas o en parajes desérticos de la Región de Atacama sin importar mayormente el impacto ambiental en el entorno. Actualmente se han reportado algunos estudios que incorporan EC de fundiciones modernas en la fabricación de morteros y hormigones con resultados satisfactorios (Nazer et al., 2012, 2013). Además, hay estudios sobre la utilización de EC como un sustituto parcial y total de áridos convencionales por EC en mezclas asfálticas para pavimentos. Hassan y Al-Jabri (2011) estudiaron el uso de EC granulada como agregado fino, hasta en un $40 \%$ de sustitución, en hormigones asfálticos en caliente y evaluados mediante ensayo Marshall. Por otra parte, Muñoz (2012) utilizó EC como un sustituto de filler (menor a $0.075 \mathrm{~mm}$, malla \#200) en proporciones de 25, 50, 75 y $100 \%$, en mezclas asfálticas evaluadas por el método Marshall. Los resultados indicaron que la EC mejora la estabilidad respecto de la mezcla control. Raposeiras et al. (2017) utilizaron una combinación de pavimento asfáltico reciclado (RAP) y EC con sustituciones de 0 a $40 \%$, y 0 a $35 \%$, respectivamente, empleando el método Marshall. Los resultados mostraron que la combinación RAP-EC otorga buenas propiedades a la mezcla asfáltica. Pérez (2017), destacando la utilización de EC como un material sustentable, indicó que ésta presentó una mayor resistencia a la desintegración en ciclos de hielo y deshielo, y una mejor adherencia con el asfalto. Pundhir et al. (2005) reportaron que la adición de EC como agregado fino en mezclas bituminosas, mejoró la trabazón y las propiedades volumétricas y mecánicas de las mezclas, sugiriendo su uso como un sustituto del agregado fino convencional. Ziari et al. (2016) evaluaron el efecto de la utilización de EC como material fino en el rendimiento de asfalto mezclado caliente, para ello estudiaron cinco mezclas de asfalto con diversos contenidos de escoria $(0,10,20,30$ y $40 \%$ en peso), obteniendo los mejores resultados con la mezcla que contenía $20 \%$ de EC.
Debido a la gravedad específica más alta de la EC en comparación con los agregados naturales, la densidad aparente aumenta con la adición de escoria (Singh y Mishra, 2016; Chetan y Sowmya, 2015). Los valores de densidad en los cuatro tipos de mezclas asfálticas utilizadas en este estudio tienden a tener el mismo comportamiento, mostrando que, al aumentar el contenido de asfalto, la densidad se incrementa hasta llegar al valor óptimo, posteriormente se observa una caída de la densidad. Por otra parte, a medida que aumenta el reemplazo del árido por EC, como se esperaba, los valores de las densidades aumentan debido a la alta densidad de la escoria. En estudios realizados por Raposeiras et al. (2017), se indicó que la presencia de la EC provoca un aumento en la densidad de las mezclas cercano al 16\%, debido al contenido de hierro incluido en su composición. Por otra parte, Pérez (2017) indicó que la EC al ser un árido más pesado podría presentar un mayor riesgo si se desprende de la superficie del pavimento, ya que tendría un potencial de destrucción mayor para vehículos circulando por la carretera.

Con relación al contenido de escoria, Ziari et al. (2015) informan que el uso de $20 \%$ de EC en mezcla de asfalto mejora la estabilidad y el rendimiento en las mezclas. Estos investigadores indican que la resistencia de las mezclas en la formación de baches disminuye sustancialmente al agregar más del 20\% de escoria de cobre. Chetan y Sowmya (2015) reportaron que, al adicionar escoria de cobre a la mezcla como reemplazo del agregado fino, la estabilidad para $20 \%$ de la EC era más alta que para 10 y $30 \%$. Por otra parte, Raposeiras et al. (2017) informaron que la incorporación de EC disminuye la estabilidad de las mezclas, obteniendo valores próximos a los obtenidos en la mezcla convencional. También se ha reportado que mezclas asfálticas preparadas con escorias de hornos de acería presentan valores más altos de estabilidad que las mezclas con agregados tradicionales, debido a ello las mezclas son más resistentes a la deformación causada por el tráfico, contribuyendo a la durabilidad del pavimento (Pérez, 2008).

Respecto de la fluencia, Raposeiras et al. (2017) informan que la adición de EC genera efectos favorables en la fluencia de las mezclas, manteniendo los valores en los límites permitidos por la norma, y cercanos a la mezcla tradicional. En cantidades elevadas de RAP se obtienen los 
valores óptimos para un 15 y $25 \%$ de EC, mientras que para cantidades bajas de RAP se recomienda utilizar 35\% de EC.

Como se ha visto, aún no se reportan estudios sobre el uso de EC como un sustituto parcial del árido grueso particularmente en el diseño de pavimentos asfálticos utilizando EC antiguas generadas por procesos de fundición con los primeros hornos de reverberos utilizados en Chile desde mediados del siglo XIX y hasta la primera década del siglo XX. Actualmente, las EC permanecen en vertederos abandonados en la costa del puerto de Caldera sin tener uso alguno (Figura 1).

El objetivo de este trabajo es evaluar mediante el método Marshall, el comportamiento de mezclas asfálticas en caliente conteniendo EC de un vertedero abandonado del siglo XIX, como un sustituto parcial del árido grueso convencional.

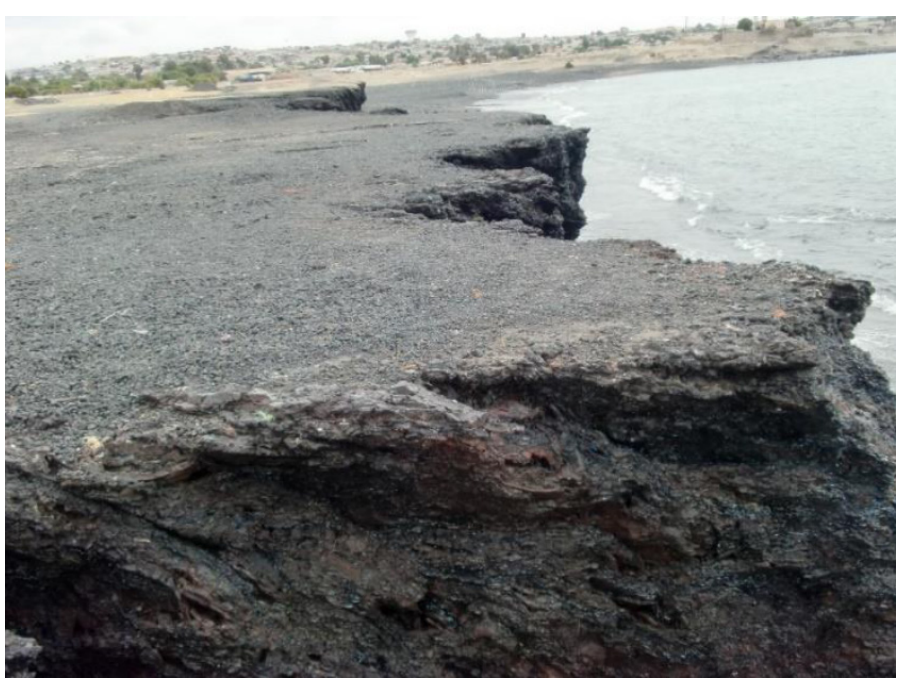

Figura 1: Vertedero de escoria de cobre abandonado en la costa de Caldera, Chile

\section{Materiales}

En el estudio realizado, se eligió el cemento asfáltico CA-24 por su grado de viscosidad absoluta, el cual se considera adecuado para el clima semiárido de la Región de Atacama, en el norte chileno. Las características del cemento asfáltico fueron determinadas en el laboratorio, verificándose el cumplimiento con los requisitos de $\mathrm{MC}$ (2012). Las características del cemento asfáltico CA-24 son mostradas en la Tabla 1.
Tabla 1: Características del cemento asfáltico CA-24

\begin{tabular}{|c|c|c|c|}
\hline Parámetro & Unidad & Valor & $\begin{array}{c}\mathrm{MC} \\
(2012)\end{array}$ \\
\hline Penetración a $25^{\circ} \mathrm{C}, 100 \mathrm{~g}, 5 \mathrm{~s}$ & $1 / 10 \mathrm{~mm}$ & 68 & $\min .40$ \\
\hline Punto de ablandamiento & ${ }^{\circ} \mathrm{C}$ & 48.5 & -- \\
\hline Viscosidad absoluta a $60^{\circ} \mathrm{C}$ & Poise & 2578 & $\geq 2400$ \\
\hline Punto de inflamación & ${ }^{\circ} \mathrm{C}$ & 363 & $\min .232$ \\
\hline Ductilidad a $25^{\circ} \mathrm{C}, \mathrm{cm} / \mathrm{min}$ & $\mathrm{cm}$ & 232 & $\min .100$ \\
\hline Ensaye de la mancha & $\%$ xilol & -30 & $\max .30$ \\
\hline Solubilidad en tricloroetileno & $\%$ & 99 & $\min .99$ \\
\hline Índice de penetración IP & - & -0.9 & $\begin{array}{l}-1.5 \mathrm{a} \\
+1.0\end{array}$ \\
\hline Pérdida por calentamiento & $\%$ & 0.08 & $\max .0 .8$ \\
\hline Penetración de residuo & $\begin{array}{l}\% \text { del } \\
\text { original }\end{array}$ & 65 & - \\
\hline $\begin{array}{l}\text { Viscosidad absoluta a } 60^{\circ} \mathrm{C} \text {, } \\
300 \mathrm{~mm} \mathrm{Hg}\end{array}$ & Poise & 6347 & - \\
\hline Ductilidad a $25^{\circ} \mathrm{C}, 5 \mathrm{~cm} / \mathrm{min}$ & $\mathrm{cm}$ & 150 & Mínimo \\
\hline Índice de durabilidad ID & - & 2.46 & $\max .3 .5$ \\
\hline $\begin{array}{l}\text { Densidad de la mezcla de } \\
\text { asfalto }\end{array}$ & $\mathrm{kg} / \mathrm{m}^{3}$ & 1010 & - \\
\hline
\end{tabular}

Se utilizó árido convencional triturado de una planta del sector Viñita Azul, modificando en el laboratorio su granulometría tomando como referencia los valores promedio (\% en peso) de la banda granulométrica semidensa que exige la especificación para la elaboración de las briquetas del ensayo Marshall. Las características físicas para los áridos de tamaños 3/4", 1/2" y 3/8" son mostradas en la Tabla 2.

Tabla 2: Parámetros físicos de los áridos convencionales según el tamaño de partículas

\begin{tabular}{|l|c|c|c|c|}
\hline \multirow{2}{*}{ Parámetro } & \multicolumn{3}{|c|}{ Gravilla } & \multirow{2}{*}{ Arena } \\
\cline { 2 - 4 } & $3 / 4 "$ & $1 / 2 ”$ & $3 / 8 "$ & \\
\hline $\begin{array}{l}\text { Densidad aparente suelta, } \\
\mathrm{kg} / \mathrm{m}^{3}\end{array}$ & 1423 & 1435 & 1459 & 1735 \\
\hline Densidad real seca, $\mathrm{kg} / \mathrm{m}^{3}$ & 2636 & 2618 & 2617 & 2607 \\
\hline Densidad neta, $\mathrm{kg} / \mathrm{m}^{3}$ & 2734 & 2718 & 2734 & 2786 \\
\hline Absorción de agua, \% & 1.36 & 1.41 & 1.65 & 2.5 \\
\hline Índice de plasticidad, \% & - & - & - & $\mathrm{NP}$ \\
\hline
\end{tabular}


La EC se recolectó de un vertedero abandonado del siglo XIX, ubicado en el sector Playa Negra en la Comuna de Caldera, Región de Atacama, Chile. Al igual que el árido convencional, la EC se clasificó por tamaño máximo de partículas necesarios para el diseño de la mezcla asfáltica. En la Tabla 3 se muestran las principales características físicas de la EC.

Tabla 3: Parámetros físicos de la EC según el tamaño de partículas

\begin{tabular}{|l|c|c|c|}
\hline \multirow{2}{*}{ Parámetro } & \multicolumn{3}{|c|}{ Gravilla } \\
\cline { 2 - 4 } & $3 / 4 "$ & $1 / 2 "$ & $3 / 8 ”$ \\
\hline $\begin{array}{l}\text { Densidad aparente suelta, } \\
\mathrm{kg} / \mathrm{m}^{3}\end{array}$ & 1606.0 & 1634.0 & 1684.0 \\
\hline Densidad real seca, $\mathrm{kg} / \mathrm{m}^{3}$ & 3240.3 & 3239.0 & 3210.7 \\
\hline Densidad neta, $\mathrm{kg} / \mathrm{m}^{3}$ & 3284.8 & 3273.9 & 3266.3 \\
\hline Absorción de agua, \% & 0.42 & 0.33 & 0.53 \\
\hline
\end{tabular}

\section{Métodos}

La toma de muestra de EC se obtuvo de cuatro calicatas de $1 \mathrm{~m}$ de profundidad aproximadamente, excavadas con retroexcavadora. La EC fue seleccionada desde las paredes y el fondo de cada calicata y posteriormente fue homogeneizada y cuarteada mediante cortador de rifle en el laboratorio de Resistencia de Materiales de Construcción de la Universidad de Atacama. La caracterización mineralógica de la muestra de EC se realizó en el laboratorio del Departamento de Geología de la Universidad Católica del Norte, para ello se utilizó el difractómetro de rayos X Siemens D5000, con goniómetro vertical Bragg-Vretano, voltaje de $40 \mathrm{kV}$ e intensidad de $30 \mathrm{~mA}$.

En este estudio se utilizó la mezcla en caliente, siendo la más usada en la Región de Atacama por su buena adherencia, trabajabilidad, resistencia al volumen de tránsito, otorgando buenas propiedades al pavimento.

Para el diseño y control de mezclas de pavimento bituminoso se utilizó el método de diseño Marshall, el cual permite determinar el contenido óptimo de asfalto para cada mezcla en función de las características del árido. En este estudio se evaluó solo el diseño de las mezclas y las probetas fueron fabricadas en el Laboratorio Regional de Vialidad de Atacama. Las briquetas fueron elaboradas con el mismo agregado pétreo y cemento asfáltico. La estabilidad de un pavimento asfáltico depende en gran medida de la graduación de los agregados, y de acuerdo con la granulometría de los áridos utilizados, se eligió la graduación semidensa establecida en MC (2014). De acuerdo con lo especificado por MC (2014), tanto para el árido triturado convencional como para la EC, se utilizó un tamaño de partículas mayor que $2.5 \mathrm{~mm}$ (\#8 ASTM), y en las siguientes proporciones: $13 \%$ de gravilla $3 / 4$ ”, $10 \%$ de gravilla de $1 / 2$ ", $27 \%$ de gravilla de $3 / 8$ " y $50 \%$ de arena.

La determinación de los parámetros Marshall se realizó en muestras en triplicado y los resultados mostrados más adelante, corresponden al promedio de estos. Los parámetros Marshall estudiados fueron densidad, huecos de aire en la mezcla, vacíos en el agregado mineral, fluencia, estabilidad, y también se determinó el óptimo de asfalto en la mezcla según la especificación 8.302.47 del MC (2012). Además, en el diseño Marshall se evaluó la mezcla asfáltica con 4.5, 5.0, 5.5, 6.0 y $6.5 \%$ de asfalto. Para el estudio, se diseñaron cuatro tipos de mezclas asfálticas con sustitución parcial de árido grueso convencional por EC, permaneciendo constante la cantidad de árido fino (550 g de arena convencional). Las sustituciones (\% en peso) evaluadas mostradas en la Tabla 4 , fueron $0 \%$ (mezcla control), 30\%, 50\% y 70\% de EC. Los ensayos se realizaron en el Laboratorio Regional de Vialidad, de acuerdo con las normas chilenas y con las especificaciones establecidas en MC (2012, 2014).

Tabla 4: Cantidad de agregado utilizada en las mezclas asfálticas

\begin{tabular}{|c|c|c|c|c|c|}
\hline $\begin{array}{c}\text { ID } \\
\text { muestra }\end{array}$ & $\begin{array}{c}\text { Propor- } \\
\text { ción }\end{array}$ & $13 \%$ & $10 \%$ & $27 \%$ & $50 \%$ \\
\hline & $\begin{array}{c}\text { EC } \\
\%\end{array}$ & $\begin{array}{c}\text { Gravilla } \\
3 / 4 " \\
\mathrm{~g}\end{array}$ & $\begin{array}{c}\text { Gravilla } \\
1 / 2 \% \\
\mathrm{~g}\end{array}$ & $\begin{array}{c}\text { Gravilla } \\
3 / 8 \% \\
\mathrm{~g}\end{array}$ & $\begin{array}{c}\text { Arena } \\
\mathrm{g}\end{array}$ \\
\hline Control & 0 & 143 & 110 & 297 & 550 \\
\hline EC-30 & 30 & 43 & 33 & 89 & 550 \\
\hline EC-50 & 50 & 72 & 55 & 149 & 550 \\
\hline EC-70 & 70 & 100 & 77 & 208 & 550 \\
\hline
\end{tabular}

En la Tabla 5 se muestran las principales características de las mezclas, cumpliendo con la especificación 5.408.201.A del MC (2014), y en la Tabla 6, la caracterización de la fracción fina, cumpliendo con la especificación 5.408.201.B del MC (2014). 
Tabla 5: Parámetros físicos de la mezcla asfáltica muestra control y muestras con EC

\begin{tabular}{|c|c|c|c|c|c|}
\hline Parámetro & Unidad & Control & EC-30 & EC-50 & EC-70 \\
\hline $\begin{array}{l}\text { Partículas } \\
\text { trituradas }\end{array}$ & $\%$ & 100 & 100 & 100 & 100 \\
\hline $\begin{array}{l}\text { Partículas } \\
\text { lajeadas }\end{array}$ & $\%$ & 1.24 & 1.62 & 3.09 & 2.60 \\
\hline $\begin{array}{l}\text { Desgaste de } \\
\text { los Angeles }\end{array}$ & $\%$ & 29 & 30 & 28 & 19 \\
\hline $\begin{array}{l}\text { Grado de } \\
\text { ensayo }\end{array}$ & grado & \multicolumn{4}{|c|}{6} \\
\hline $\begin{array}{l}\text { Índice de } \\
\text { plasticidad }\end{array}$ & \multicolumn{5}{|c|}{ NP } \\
\hline $\begin{array}{l}\text { Sales solubles } \\
\text { totales }\end{array}$ & \multicolumn{5}{|c|}{ no se detectan } \\
\hline $\begin{array}{l}\text { Desintegración } \\
\text { por sulfato de } \\
\text { sodio }\end{array}$ & $\%$ & 1.21 & 1.05 & 0.92 & 0.58 \\
\hline $\begin{array}{l}\text { Adherencia } \\
\text { con CA-24 }\end{array}$ & $\%$ & \multicolumn{4}{|c|}{+95} \\
\hline $\begin{array}{l}\text { Método } \\
\text { estático } \\
\text { Riedel-Weber } \\
\text { (escala 0-10) } \\
\end{array}$ & grado & \multicolumn{4}{|c|}{$2-7$} \\
\hline $\begin{array}{l}\text { Densidad } \\
\text { máxima de la } \\
\text { mezcla suelta }\end{array}$ & $\mathrm{kg} / \mathrm{m}^{3}$ & \multicolumn{4}{|c|}{2456} \\
\hline $\begin{array}{l}\text { Asfalto } \\
\text { referido al } \\
\text { agregado }\end{array}$ & $\%$ & \multicolumn{4}{|c|}{5.5} \\
\hline $\begin{array}{l}\text { Cantidad de } \\
\text { muestras }\end{array}$ & \multicolumn{5}{|c|}{3} \\
\hline $\begin{array}{l}\text { Tipo de } \\
\text { recipiente }\end{array}$ & \multicolumn{5}{|c|}{ A } \\
\hline $\begin{array}{l}\text { Densidad del } \\
\text { asfalto }\end{array}$ & $\mathrm{kg} / \mathrm{m}^{3}$ & \multicolumn{4}{|c|}{1010} \\
\hline $\begin{array}{l}\text { Densidad } \\
\text { efectiva }\end{array}$ & $\mathrm{kg} / \mathrm{m}^{3}$ & 2665.92 & 2667.20 & 2831.02 & 2798.48 \\
\hline $\begin{array}{l}\text { Densidad } \\
\text { real seca } \\
\text { ponderada }\end{array}$ & $\mathrm{kg} / \mathrm{m}^{3}$ & 2614.63 & 2704.94 & 2765.14 & 2825.34 \\
\hline
\end{tabular}

Tabla 6: Parámetros de la fracción fina de áridos

\begin{tabular}{|l|c|c|}
\hline Parámetro & Unidad & Resultado \\
\hline Índice de plasticidad & $\%$ & NP \\
\hline Sales solubles & $\%$ & no se detectan \\
\hline Equivalente de arena & $\%$ & 78.62 \\
\hline Procedimiento agitación & - & mecánica \\
\hline $\begin{array}{l}\text { Adherencia con CA-24: Riedel- } \\
\text { Weber }(0-10)\end{array}$ & grado & $2-7$ \\
\hline
\end{tabular}

Por otra parte, el contenido óptimo de asfalto y las especificaciones para las diferentes muestras, y el diseño de la mezcla Marshall de la muestra control es presentado en las Tablas 7 y 8 , respectivamente.
Tabla 7: Contenido óptimo de asfalto para las diferentes mezclas asfálticas

\begin{tabular}{|l|c|c|c|c|}
\hline $\begin{array}{c}\text { Contenido óptimo de } \\
\text { asfalto, } \\
\text { \% ref. al agregado }\end{array}$ & Control & EC-30 & EC-50 & EC-70 \\
\hline $\begin{array}{l}\text { Asfalto para máxima } \\
\text { densidad }\end{array}$ & 6.5 & 6.5 & 6.0 & 6.5 \\
\hline $\begin{array}{l}\text { Asfalto para máxima } \\
\text { estabilidad }\end{array}$ & 5.5 & 5.5 & 6.0 & 6.5 \\
\hline $\begin{array}{l}\text { Asfalto para 5\% de } \\
\text { huecos de aire }\end{array}$ & 5.5 & 5.0 & 5.8 & 5.5 \\
\hline Promedio y tolerancia & $\begin{array}{l}5.8 \pm \\
0.3 \%\end{array}$ & $\begin{array}{l}5.7 \pm \\
0.3 \%\end{array}$ & $\begin{array}{l}5.9 \pm \\
0.3 \%\end{array}$ & $\begin{array}{c}6.2 \pm \\
0.3 \%\end{array}$ \\
\hline
\end{tabular}

Tabla 8: Características de la mezcla asfáltica de la muestra control

\begin{tabular}{|l|c|c|c|c|c|c|c|}
\hline Parámetro & Unidad & \multicolumn{6}{|c|}{ Mezcla asfáltica } \\
\hline CA 60-80 & $\begin{array}{c}\% \\
\text { asfalto }\end{array}$ & 4.5 & 5.0 & 5.5 & 6.0 & 6.5 & 7.0 \\
\hline Densidad & $\mathrm{kg} / \mathrm{m}^{3}$ & 2295.6 & 2314.0 & 2332.9 & 2335.9 & 2355.2 & 2349.2 \\
\hline DMM $^{*}$ & $\mathrm{~kg} / \mathrm{m}^{3}$ & 2490.1 & 2472.9 & 2456.0 & 2439.5 & 2423.4 & 2407.7 \\
\hline $\begin{array}{l}\text { Huecos } \\
\text { Va }\end{array}$ & $\%$ & 7.81 & 6.42 & 5.01 & 4.25 & 2.81 & 2.43 \\
\hline VAM** & $\%$ & 15.98 & 15.71 & 15.43 & 15.72 & 15.42 & 16.03 \\
\hline Fluencia & $\begin{array}{c}0.25 \\
\mathrm{~mm}\end{array}$ & 10.3 & 15.5 & 10.7 & 13.2 & 9.8 & 14.4 \\
\hline $\begin{array}{l}\text { Estabili- } \\
\text { dad }\end{array}$ & $\mathrm{N}$ & 13021 & 12901 & 14594 & 12492 & 12663 & 12921 \\
\hline
\end{tabular}

* Densidad máxima de la mezcla sin compactar

** Porcentaje de vacíos en el agregado mineral

\section{Resultados}

\section{Caracterización mineralógica de la EC}

En la Tabla 9 se puede observar que la EC está compuesta principalmente por clinopiroxeno, silicato de hierro, magnesio-ferrita y titanio-magnetita.

Tabla 9: Características mineralógicas de la EC

\begin{tabular}{|l|c|c|}
\hline Compuesto & Fórmula & $\%$ \\
\hline Clinopiroxeno & $\left(\mathrm{Mg}_{0.6} \mathrm{Fe}_{0.2} \mathrm{Al}_{0.2}\right) \mathrm{Ca}\left(\mathrm{Si}_{1.5} \mathrm{Al}_{0.5}\right) \mathrm{O}_{6}$ & 33.2 \\
\hline Silicato de hierro & $\mathrm{Fe}_{2} \mathrm{SiO}_{4}$ & 16.6 \\
\hline Magnesio-ferrita & $\mathrm{MgFe}_{2} \mathrm{O}_{4}$ & 13.8 \\
\hline Titanio-magnetita & $\mathrm{Fe}_{2} \mathrm{TiO}_{4}$ & 13.8 \\
\hline Óxido de bario y titanio & $\mathrm{BaTiO}_{3}$ & 11.8 \\
\hline Westita & $\mathrm{FeO}$ & 5.6 \\
\hline Pigeonita & $\left(\mathrm{Mg}_{0.39} \mathrm{Fe}_{0.52} \mathrm{Ca}_{0.09}\right) \mathrm{SiO}_{3}$ & 4.8 \\
\hline Nantokita & $\mathrm{CuCl}$ & 0.3 \\
\hline Cuprita & $\mathrm{Cu}_{2} \mathrm{O}$ & 0.1 \\
\hline
\end{tabular}




\section{Parámetros Marshall}

En la Figura 2, se pueden observar los resultados promedios de la densidad en función de las variables contenido de asfalto y contenido de EC como sustituto del árido convencional. Para todas las muestras conteniendo EC se observa que poseen mayor densidad que la muestra control ( $0 \%$ EC). Como es natural, se aprecia que a medida que aumenta la cantidad de asfalto y el contenido de EC en la mezcla, en general aumenta su densidad. La mayor densidad alcanzada por todas las mezclas conteniendo EC en comparación con el control, se explica por la mayor densidad que posee la EC respecto del árido convencional.

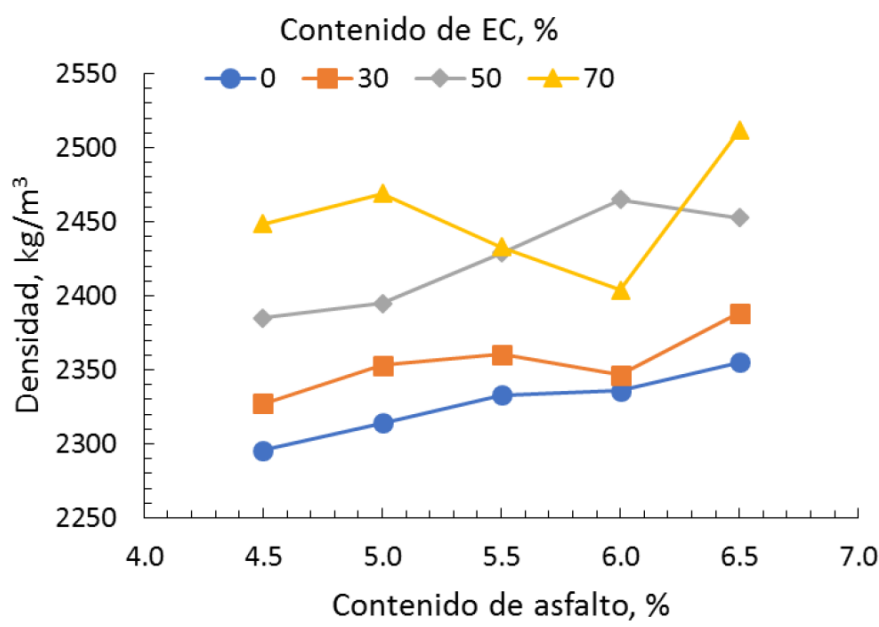

Figura 2: Densidad de las mezclas en función del \% de asfalto y contenido de EC

En la Figura 3 puede observarse que todas las muestras que contienen EC presentan estabilidades de las mezclas inferiores al control. De acuerdo con la especificación de la tabla 5.408.203.A del MC (2014), la muestra control y la muestra EC-70 cumplirían el requisito de mezcla asfáltica para una carpeta de rodadura (9000 N mínimo) para todos los contenidos de asfalto estudiados, mientras que la muestra EC-30 cumple con el requisito de estabilidad para contenidos de asfalto de 5.0,5.5 y 6.5\%. Un comportamiento similar presenta la muestra EC-50 cumpliendo para todos los contenidos de asfalto, excepto para $5 \%$ de asfalto. Sin embargo, se puede observar que todas las muestras ensayadas cumplen con la especificación de estabilidad para el tipo de mezcla asfáltica para una base gruesa $(6000$ $\mathrm{N}$ mínimo). En general, al incrementar el contenido de asfalto la estabilidad aumenta, cohesionando las partículas del árido hasta llegar al óptimo en torno a 5.5\%.
Contenido de EC, \%

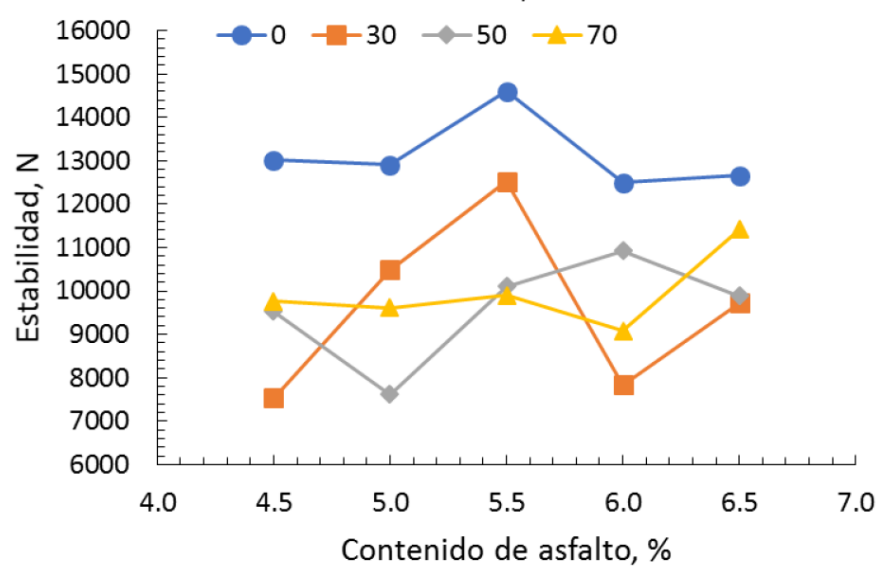

Figura 3: Estabilidad de las mezclas en función del \% de asfalto y contenido de EC

En la Figura 4 se puede observar que en general todas las mezclas asfálticas estudiadas cumplen con la fluencia establecida en la especificación de la tabla 5.408.203.A del MC (2014), al situarse en el rango de 8 a $16 \mathrm{~mm}$, exceptuando la muestra EC-70 con el contenido de asfalto de $6.5 \%$ la que se ubica levemente por debajo del mínimo.

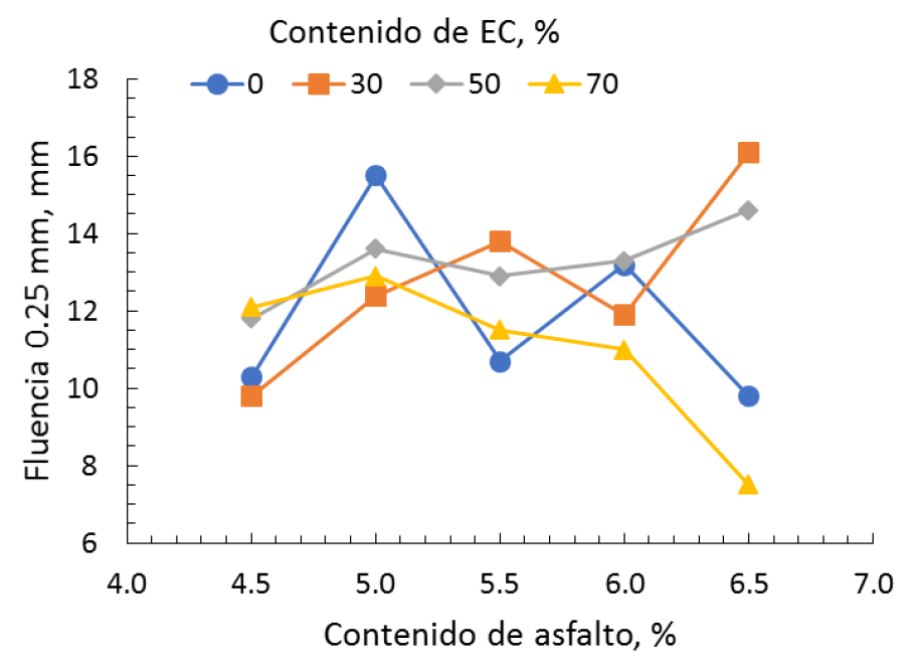

Figura 4: Fluencia de las mezclas en función del \% de asfalto y contenido de EC

En la Figura 5, como es natural, se confirma que a mayor presencia de asfalto y de EC en la mezcla tiende a disminuir el porcentaje de huecos con aire en las briquetas. Comparando los valores de las curvas con los requisitos establecidos en la tabla 5.408.203.A del MC (2014), todas las muestras cumplen con las especificaciones para una base asfáltica gruesa (5 a 10\% de huecos) con contenidos de asfalto de 4.5 y $5.0 \%$, mientras que las muestras control, 
EC-30 y EC-70 cumplen para una capa intermedia (3 a 8\% de huecos) con contenidos de asfalto en el rango de 4.5 a $6.0 \%$. Además, las muestras EC-30 y EC-70 cumplen para una carpeta de rodadura ( 4 a $6 \%$ de huecos) con contenidos de asfalto de 5.0 y $6.0 \%$.

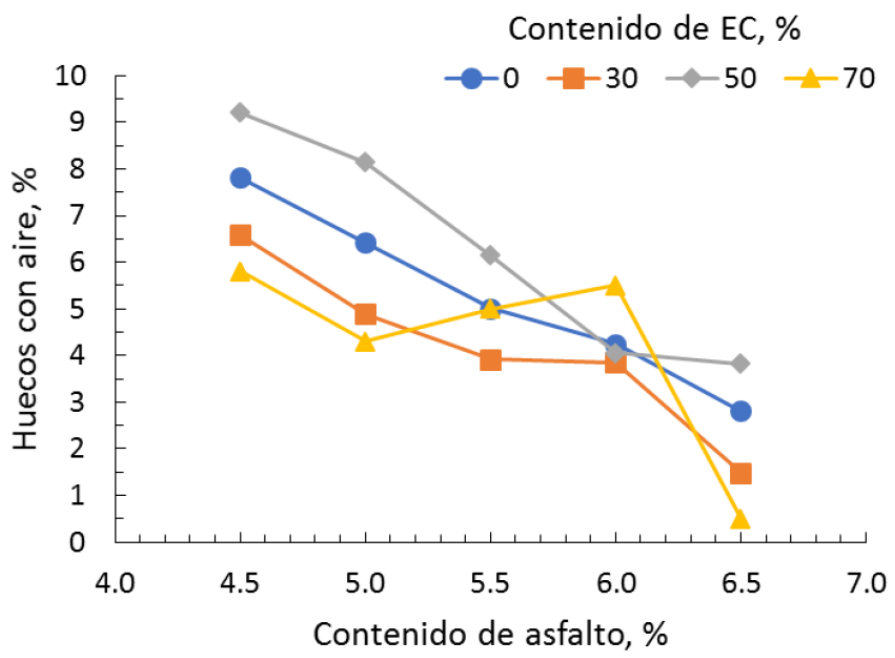

Figura 5: Contenido de huecos en las mezclas en función del \% de asfalto y contenido de EC

En la Figura 6, se observa que todas las mezclas asfálticas estudiadas cumplen con el requisito de la especificación 5.408.203.B del MC (2014) para el contenido de vacíos de los agregados minerales, el cual es de $14 \%$ para el tamaño máximo de partículas, que para este estudio fue de $20 \mathrm{~mm}$.

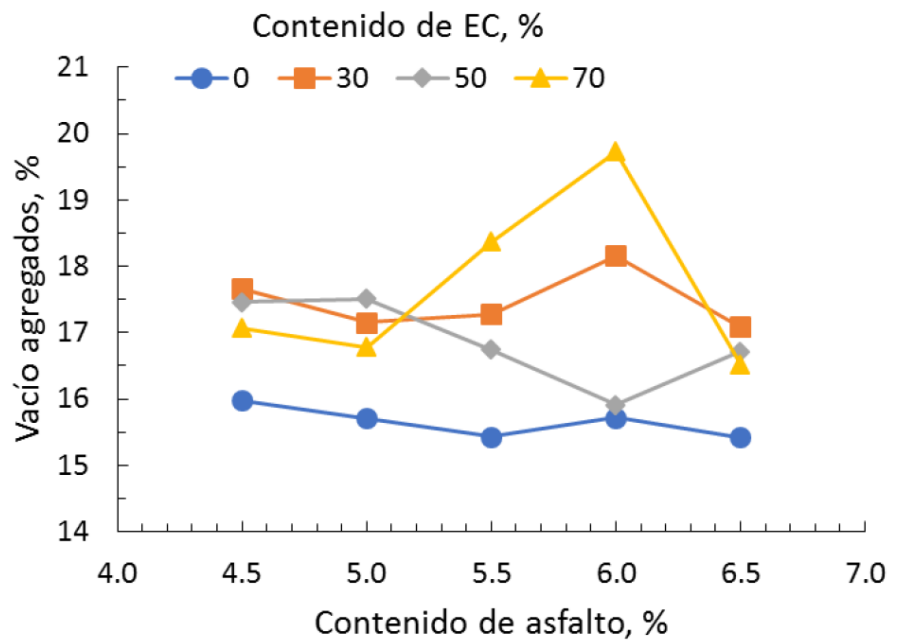

Figura 6: Vacío del agregado mineral en la mezcla en función del $\%$ de asfalto y contenido de EC

\section{Conclusiones}

Como conclusión general de este estudio, se puede señalar que:
Todas las muestras con sustituciones parciales de EC tienen mayor densidad que la muestra control. Por el contrario, la estabilidad se ve reducida en todas las muestras que contienen EC en comparación con la muestra que sirvió de control, sin embargo, cumplen con la especificación de estabilidad para el tipo de base asfáltica gruesa definida en el Manual de Carreteras (MC, 2014). La fluencia también es un parámetro de cumplimiento con los requisitos del Manual de Carreteras (MC, 2014) en todas las muestras ensayadas.

En relación con el contenido de huecos con aire, todas las muestras cumplen con las especificaciones para base asfáltica gruesa para los contenidos de asfalto de $4.5 \mathrm{y}$ $5.0 \%$; mientras que las muestras EC-30 y EC-70 cumplen tanto para una capa intermedia con contenidos de asfalto en el rango de 4.5 a $6.0 \%$, como para una carpeta de rodadura para contenidos de asfalto de 5.0 y $6.0 \%$.

Respecto del contenido de vacío de los agregados minerales, todas las mezclas asfálticas estudiadas cumplen con el requisito de la especificación del Manual de Carreteras (MC, 2014).

$\mathrm{Al}$ diseñar un pavimento asfáltico con sustitución parcial de agregados convencionales por EC, los resultados sugieren su utilización hasta en un $30 \%$ de su contenido pétreo en la fracción gruesa, ya que una sustitución mayor, demandaría un aumento de la cantidad de asfalto requerido para alcanzar el diseño óptimo.

La utilización de pasivos ambientales mineros, como las EC estudiadas en mezclas asfálticas en caliente, contribuyen a mejorar las condiciones ambientales y la demanda de áridos en zonas semi desérticas como la Región de Atacama en Chile.

\section{Agradecimientos}

Los autores agradecen al personal del Laboratorio Regional de Vialidad Atacama, por contribuir con el equipamiento en la realización de los ensayos.

\section{Referencias}

Chetan, M.K. and Sowmya, N.J. (2015). Utilization of copper slag in bituminous concrete with a stone dust and fly ash as a filler material. International Journal for Research in Applied Science \& Engineering Technology 3(VI), 425-431 
Hassan, H.F. and Al-Jabri, K. (2011). Laboratory evaluation of hot-mix asphalt concrete containing copper slag aggregate. Journal of Materials in Civil Engineering 23(6), 879-885

MC (2014). Especificaciones técnicas generales de construcción. Manual de Carreteras, volumen 5, Dirección de Vialidad, Ministerio de Obras Públicas de Chile

MC (2012). Especificaciones y métodos de muestreo, ensaye y control. Manual de Carreteras, volumen 8, Dirección de Vialidad, Ministerio de Obras Públicas de Chile

Muñoz, H. (2012). Análisis de pavimentación (MDC-3) mejoradas con escorias (no ferrosas) de fundición de cobre (ensayo Marshall INV-E 748-07). Trabajo de grado Ingeniero Civil, Universidad Militar Nueva Granada, Bogotá, Colombia Nazer, A., Pavez, O. and Rojas, F. (2012). Use of copper slag in cement mortar. Revista Escola de Minas 65(1), 87-91

Nazer, A., Pavez, O. and Toledo, I. (2013). Effect of type cement on the mechanical strength of copper slag mortars. Revista Escola de Minas 66(1), 85-90

Pérez, Y. (2017). Utilización de escoria de cobre de CODELCO Ventanas como agregado pétreo para tratamientos superficiales asfálticos simples en la Región de Valparaíso. Memoria Ingeniero Civil, Universidad Técnica Federico Santa María, Valparaíso, Chile
Pérez, E. (2008). Evaluación de las escorias de horno como agregado en mezclas asfálticas. Trabajo de graduación Ingeniero Civil, Universidad de San Carlos de Guatemala, Guatemala

Pundhir, N.K.S., Kamaraj, C. and Nanda, P.K. (2005). Use of copper slag as construction material in bituminous pavements. Journal of Scientific \& Industrial Research 64, 997-1002

Raposeiras, A., Movilla, D., Vargas, A., Bilbao, R. and Cifuentes, C. (2017). Evaluación de rigidez Marshall, tracción indirecta y módulo resiliente en mezclas asfálticas con RAP y escoria de cobre. Revista Ingeniería de Construcción 32(1), 15-24

Singh, S. and Mishra, A.K. (2016). Utilization of copper slag and stone dust in bituminous pavement with hydrated lime as filler material. International Journal of Advance Research in Science and Engineering 5(4), 237-250

Ziari, H., Moniri, A., Ayazi, M.J. and Nakhaei, M. (2016). Investigation of rutting performance of WMA mixtures containing copper slag. International Journal of Transportation Engineering 3(3), 227-235 